\title{
Effects of consistent or deviant modeling cues on the adoption of a self-imposed standard'
}

JAE H. HILL and ROBERT M. LIEBERT, Vanderbilt University, Nashville, Tenn. 37203

This study investigated the effects of consistent or deviant modeling cues exhibited by $0,1,2$, or 3 successively presented models upon children's adherence to an explicitly stated stringent standard which they had been previously taught. Ss who observed deviant social models were less likely to precisely adhere to the stated rule than those in the no-model control group, while those who observed consistent models were more likely to do so. Moreover, the pattern of results suggests that the influence of modeling cues in this situation is one of relatively precise matching rather than a generalized inhibition or disinhibition effect.

An informational analysis of the effects of modeling cues (Allen \& Liebert, in press) implies that direct instruction should be both strengthened by consistent modeling and weakened by deviant modeling. Exploration of this hypothesis, only partially tested by Allen and Liebert and supported by earlier research which failed to include a trained control group (McMains \& Liebert, 1968), was the primary purpose of the present study. Additionally, the data were analyzed so as to discriminate between disinhibition and modeling effects (cf. Bandura \& Walters, 1963) and further clarification of the effects of multiple modeling was sought.

\section{METHOD}

The Ss were 21 boys and 21 girls ( $9-10$ years of age) from a public school. An equal number of Ss from each sex were randomly assigned to each of seven treatments, representing the cells of a 3 (number of models) by 2 (consistent or deviant modeling) factorial experiment plus a single control group (Winer, 1962, p. 263). Three adult males served as the models for the six experimental groups, each serving an equal number of times in each modeling position for each treatment group. An adult female served as the training agent for all Ss.

The task was a bowling game (fully described by Allen \& Liebert, in press) which appears to require skill but was, in fact, programmed to yield on 18 trial sequences including five

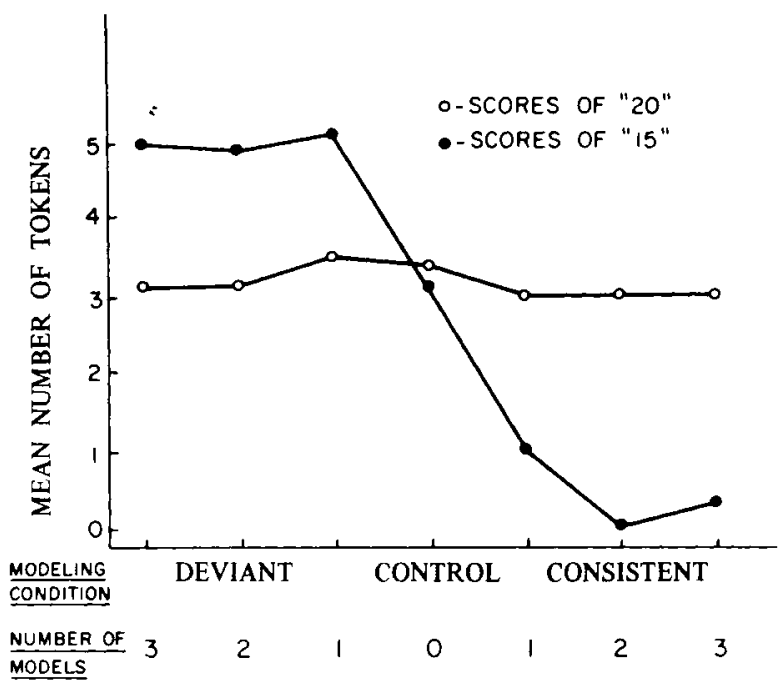

Fig. 1. Mean self-reward for scores of " 15 " and " 20 " in all groups. instances each of the scores " 5 ," "10," and " 15 ," and three instances of " 20 ." The training agent first directly instructed each child to take a reward token only for scores of " 20 ," explaining that the reward tokens could be exchanged for prizes and "the more tokens you have the better your prize will be." The $S$ then observed either zero, one, two, or three models perform the game and, as each finished, he took his tokens and left the trailer. The number of trials modeled for the Ss was constant across all groups. Thus, in the One Model conditions the model bowled 18 trials; in the Two Models conditions each model bowled nine trials; and in the Three Models conditions each model bowled six trials. Each child was then left alone to play the game while having free access to the reward tokens. All of the models observed by a given $\mathrm{S}$ were consistent with each other. Models in the deviant condition took tokens for all scores of " 15 " and " 20 ," while consistent models took tokens for every " 20 " and for no other scores.

\section{RESULTS}

An inspection of the data revealed no tendency for sex differences and consequently the data for the two sexes were combined for all analyses. Furthermore, an inspection of the mean number of self-administered tokens for scores of " 5 " and " 10 " revealed no differences for these two scores, which were therefore also combined for analysis.

As seen in Fig. 1, the six groups comprising the factorial part of the experimental design did not differ with respect to the mean number of tokens self-administered in the presence of the score "20." The 3 by 2 analysis of variance yielded no significant Fs nor did the Control group differ from the six factorial groups on this measure $(t=.82)$.

It is also apparent in Fig. 1 that the six groups in the factorial section did differ with respect to the mean number of tokens taken for scores of "15." The analysis of variance revealed a highly significant effect for consistent vs deviant modeling $(F=207.53, p<.000001)$ and no other significant Fs. Since there was no main effect or interaction for number of models on this measure, the groups were collapsed for further analyses of the "15" data. These showed that Ss in the

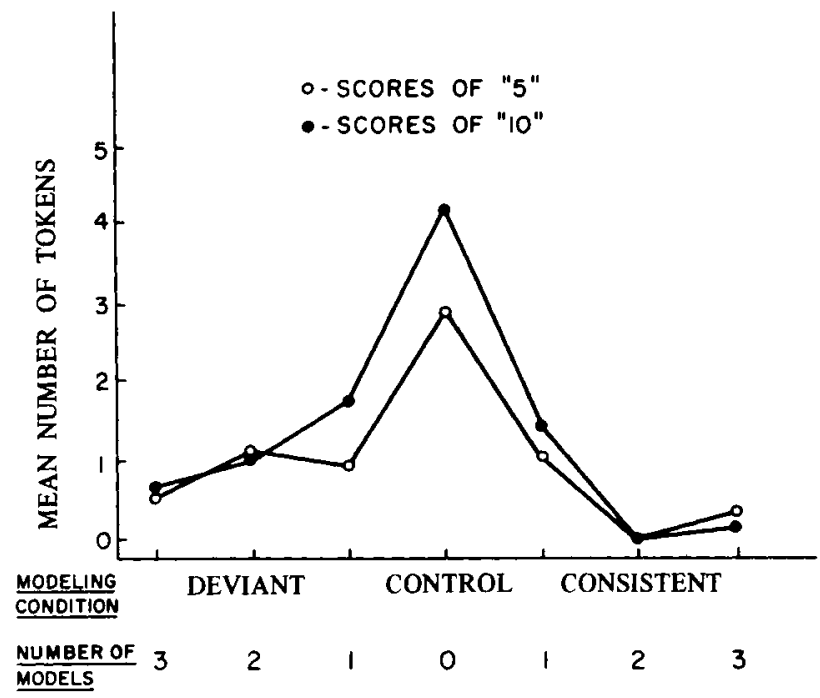

Fig. 2. Mean self-reward for scores of " 5 " and " 10 " in all groups. 
Consistent groups took fewer tokens for scores of " 15 " than the children in the Control group $(t=3.58, p<.001$, one-tailed), while Ss in the Deviant groups took more tokens than their counterparts in the Control group on this measure $(t=3.09, p<.005$, one-tailed). The 3 by 2 analy sis of variance for the scores " 5 " and " 10 " revealed no significant Fs. However, as seen in Fig. 2, the children in the Control group took more tokens in the presence of these two scores than did the $S s$ in the Experimental groups $(t=4.15, p<.005$, two-tailed).

\section{DISCUSSION}

As predicted, when the modeled behavior was consistent with the direct instruction which they received and the models whom they saw, Ss in these groups self-administered token with the direct instruction which they received and the models whom they say, Ss in these groups self-administered token rewards for virtually every score of " 20 ," but, as seen in Fig. 1, virtually never for scores of "15." In fact, despite a powerful incentive, more than $77 \%$ of the children in the consistent modeling groups self-administered tokens for every score of " 20 " and for no other score.

In sharp contrast to the consistent modeling groups, Ss who observed a modeled standard which was inconsistent with the stated rule almost uniformly lowered their own standards, thus emulating the model(s) and violating direct instruction. As seen in Fig. 1, Ss in these groups rewarded themselves for scores of "15" substantially more often than Ss in either the consistent modeling or control groups. In fact, no child exposed to deviant modeling restricted himself to scores of "20." But perhaps even more interesting is the behavior of Ss in the deviant modeling groups with respect to scores of " 5 " and "10". Recall that both the direct instruction which these children received and the deviant models whom they saw suggested that these lowest scores were not deserving of self-reward. Ss exposed to this convergence of cues rewarded themselves for scores of "15" (as modeled) significantly more than Ss in the no modeling group, but significantly less for scores of " 5 " and " 10 ." Thus, as in the consistent modeling groups, the influence of modeling cues appears to be one of quite precise matching rather than a generalized inhibition or disinhibition effect.

No significant effects were found for the number of models employed. However, as Fig. 1 illustrates for the critical scores of "15," the failure to find differences for this variable may be due to a "floor" and "ceiling" effect in the present experimental situation. The presentation of a single model was sufficient to produce almost complete acceptance of scores of " 15 " in the deviant modeling conditions and almost complete avoidance of these scores in the consistent modeling conditions.

\section{REFERENCES}

ALLEN, M. K., \& LIEBERT, R. M. Effects of live and symbolic deviant modeling cues on adoption of a previously learned standard. Journal of Personality \& Social Psychology, in press.

BANDURA, A., \& WALTERS, R. H. Social learning and personality development. New York: Holt, Rinehart \& Winston, 1963.

McMAINS, M. J., \& LIEBERT, R. M. The influence of discrepancies between successively modeled self-reward criteria on the adoption of a self-imposed standard. Journal of Personality \& Social Psychology, $1968,8,166-171$.

WINER, B. J. Statistical principles in experimental design. New York: McGraw-Hill, 1962.

$$
\text { NOTE }
$$

1. This study was supported, in part, by United States Office of Education Grant No. OEG-2-7-070002-3005 and by National Institute of Child Health and Human Development Grant No. HD 03573-01. Grateful acknowledgement is made to the administrative of ficials and teachers of the Metropolitan Nashville School System, without whose generous cooperation this research would not have been possible. The assistance of Margaret Hanratty, Richard King, Frances Owen, Robert Robbins, and Louise White is also acknowledged. 